\title{
Application of Fuzzy PID Control in Motor Test
}

\author{
Shuai Zhang \\ Shanghai Stock Exchange Technology Co., Ltd., Huajing Road No.1, Pudong Shanghai. China \\ shuaizhang@sse.com.cn
}

Keywords: PID control; fuzzy control; motor test.

\begin{abstract}
According to the disadvantage of the traditional PID control, a fuzzy adaptive PID controller is established on the basis of the traditional PID controller. This dissertation proposes the application of the fuzzy PID controller in motor test systems. Then it discusses the design of fuzzy PID controller in details, and it also shows the respective curves of the dynamic response using traditional PID controller and fuzzy PID controller. Simulation results show that by using fuzzy PID controller, the faster response and the smaller excess can be obtained.
\end{abstract}

\section{Introduction}

The motor needs to be tested. The tests are often required to adjust the motor running to a specific operating point, such as adjusting the motor to run to a target torque, speed or power. Then the corresponding parameters of motor are measured by a variety of instrumentation. Ultimately, the characteristic curve of the motor can be drawn[1]. These parameters are obtained through the no-load test, load test, temperature test, torque test and other tests. Therefore, the fine motor test system could not only measure the motor operating parameters such as torque, speed and power but also control the motor running to a operating point quickly and accurately.

\section{The Traditional PID Controller}

Traditional motor control utilizes the PI or PID control. There is often a good control performance for the specific model of motor. But for another model of the motor, if the mechanical time constant have a apparent change, then the adjusting performance of PID or PI controller will be far from ideal. There will be a large overshoot or slow response.

In the process of motor test, in order to get a good repeatability and high accuracy test results, the adjustment process of the operating point must be as short as possible to minimize the heating of the tested motor[2]. In addition, the operating point changes must be monotonically decreasing or increasing due to the hysteresis of the motor magnetic circuit. The phenomenon that overshooting or operating point changing back and forth should not emerge. Thus, PID or PI controller that relies on mathematical models can not meet the test requirements of precision motors.

Based on the considering above, this dissertation proposes a fuzzy controller of online adjusting PID parameters[3]. It is the method that combining fuzzy control and traditional PID control. Parameter adjustment rules deposited in the storage unit of the processor. According to the situation, parameters are auto-adjusted on-line based on parameter adjustment rule deposited. It is applicated in the motor test system based on the driving system and get good results.

\section{The Fuzzy Self-adaptive PID Controller}

In this dissertation, the method is illustrated that designing the fuzzy self-adaptive PID controller in the speed regulating. In the speed regulating,speed regulation controller utilizes fuzzy self-adaptive PID controller in order to compensate for the shortcomings of PID regulator coefficients $k_{p}, k_{i}, k_{d}$ setting difficulty. 


\subsection{The Principle of Fuzzy Self-adaptive PID Controller}

Fuzzy adaptive PID controller of which input is error e and error changing rate $\Delta \mathrm{e}$ could meet the requirements of $\mathrm{e}$ and $\Delta \mathrm{e}$ to PID parameter self-adjusting at different moments. The fuzzy self-adaptive PID controller utilizes fuzzy control rules to modify the PID parameters online, and its structure is shown in Figure1

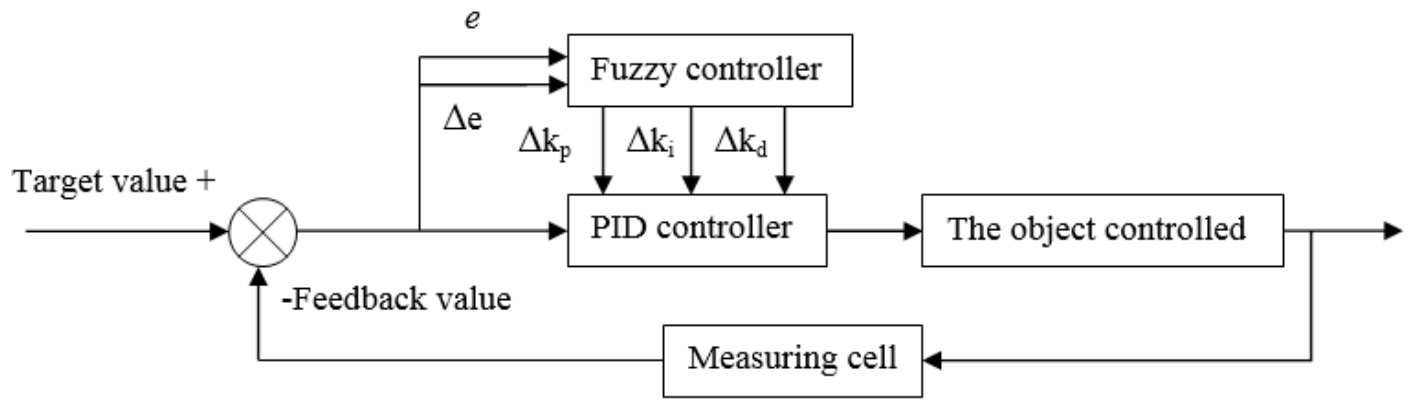

Figure 1. The fuzzy self-adaptive PID controller chart

The schematic ${ }^{[4]}$ of fuzzy PID correction online is shown in Figure 2. The adjusting of PID parameter must take the relationship of the three parameters at the different moment into account, which make controlled object achieve the best combination of static and dynamic performance. Then the fuzzy control rule table is established.

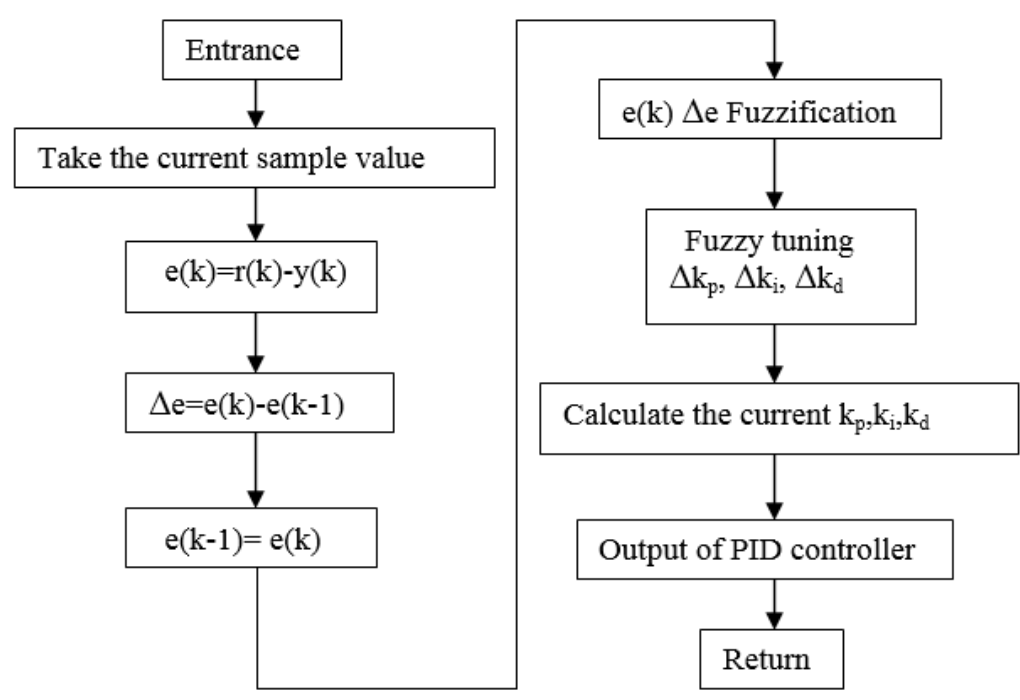

Figure 2. The fuzzy PID controller working chart

\subsection{The Fuzzy Rule Table}

For illustration, this dissertation order parameters kp for example to illustrate the creation of fuzzy control rule table. The number of fuzzy partition of input and output variables of fuzzy PID controller are taken as 7 , It is quantified to the domain of [-7,+7]. The fuzzy subset of e, $\Delta \mathrm{e}, \Delta \mathrm{k}_{\mathrm{p}}, \Delta \mathrm{k}_{\mathrm{i}}, \Delta \mathrm{k}_{\mathrm{d}}$. Chooses the following set of words: $\{\mathrm{NB}, \mathrm{NM}, \mathrm{NS}, \mathrm{NZ}, \mathrm{ZE}, \mathrm{PZ}, \mathrm{PS}, \mathrm{PM}, \mathrm{PB}\}$, that respectively is, negative big, negative medium, negative small, zero, positive small, positive medium, positive big. The triangular membership function curves are selected. The curve close to the target point should have a higher resolution in order to meet the control accuracy requirements. So the membership function curves of input and output variable of fuzzy PID controller are selected as shown in Figure 3: 


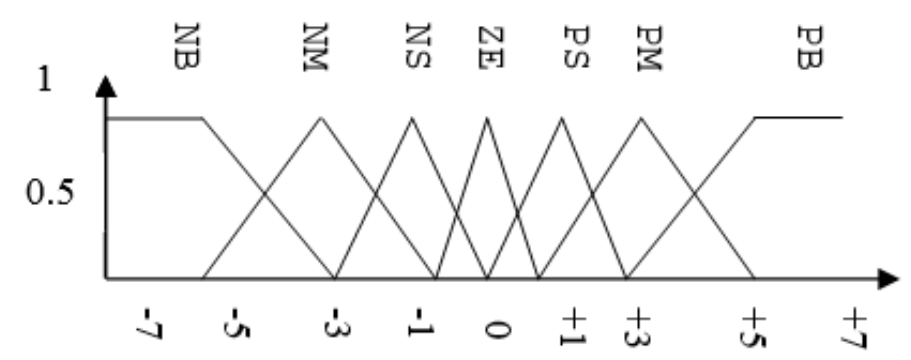

Figure 3. The membership function curves of e, $\Delta \mathrm{e}, \Delta \mathrm{kp}, \Delta \mathrm{ki}, \Delta \mathrm{kd}$

The fuzzification of input and output variables can be achieved according to membership function curves of e, $\Delta \mathrm{e}$ and $\Delta \mathrm{k}_{\mathrm{p}}$.

The system adopts two-dimensional input and one-dimensional output, so using logic statements as follows

If $\mathrm{e}$ is $\mathrm{A}$ AND $\triangle \mathrm{e}$ is $\mathrm{B}$ THEN $\triangle \mathrm{u}$ is $\mathrm{C}$

$\mathrm{A}, \mathrm{B}$, and $\mathrm{C}$ is the language variable of domain $\{\mathrm{NB}, \mathrm{NM}, \mathrm{NS}, \mathrm{NZ}, \mathrm{ZE}, \mathrm{PZ}, \mathrm{PS}, \mathrm{PM}, \mathrm{PB}$.

e.g. If e is NS AND $\triangle \mathrm{e}$ is PB THEN $\Delta \mathrm{k}_{\mathrm{p}}$ is NS

That is the deviation of input e is negative small. And the rate of changing of the deviation of input $\Delta \mathrm{e}$ is positive big. Then the variation of control value $\Delta \mathrm{k}_{\mathrm{p}}$ is negative small.

Write the specific fuzzy control state table. The fuzzy control rule table is the core content of fuzzy control. It is concluded on the basis of actual experience. Fuzzy control rule table is clear and intuitive in description of the fuzzy control rules. The following is the fuzzy control rule table of $\Delta \mathrm{k}_{\mathrm{p}}$ during the operation of motor. It is shown in Table 1:

Table 1. The fuzzy control rule table of $\Delta \mathrm{kp}$

\begin{tabular}{|c|c|c|c|c|c|c|c|}
\hline$e$ & NB & NM & NS & ZE & PS & PM & PB \\
\hline NB & PB & PB & PM & PM & PS & ZE & ZE \\
\hline NM & PB & PB & PM & PS & PS & ZE & NS \\
\hline NS & PM & PM & PM & PS & ZE & NS & NS \\
\hline ZE & PM & PM & PS & ZE & NS & NM & NM \\
\hline PM & PS & PS & ZE & NS & NS & NM & NM \\
\hline PB & ZE & ZE & NS & NM & NM & NM & NB \\
\hline
\end{tabular}

In the same way, the control rule table of $\Delta \mathrm{k}_{\mathrm{i}}$ and $\Delta \mathrm{k}_{\mathrm{d}}$ are shown in Table 2, Table 3.

Table 2. The fuzzy control rule table of $\Delta \mathrm{ki}$

\begin{tabular}{|c|c|c|c|c|c|c|c|}
\hline $\begin{array}{c}\Delta \mathrm{e} \\
\mathrm{NB}\end{array}$ & $\mathrm{NB}$ & $\mathrm{NM}$ & $\mathrm{NS}$ & $\mathrm{ZE}$ & $\mathrm{PS}$ & $\mathrm{PM}$ & $\mathrm{PB}$ \\
\hline NM & NB & NB & NM & NM & NS & ZE & ZE \\
\hline NS & NB & NM & NS & NS & ZE & PS & NS \\
\hline ZE & NM & NM & NS & ZE & PS & PM & PM \\
\hline PS & NM & NS & ZE & PS & PS & PM & PB \\
\hline PM & ZE & ZE & PS & PS & PM & PB & PB \\
\hline PB & ZE & ZE & PS & PM & PM & PB & PB \\
\hline
\end{tabular}


Table 3. The fuzzy control rule table of $\Delta \mathrm{kd}$

\begin{tabular}{|c|c|c|c|c|c|c|c|}
\hline$\Delta \mathrm{e}$ & NB & NM & NS & ZE & PS & PM & PB \\
\hline NB & PS & NS & NB & NB & NB & NM & PS \\
\hline NM & PS & NS & NB & NM & NM & NS & ZE \\
\hline NS & ZE & NS & NM & NM & NS & NS & ZE \\
\hline ZE & ZE & NS & NS & NS & NS & NS & ZE \\
\hline PS & ZE & ZE & ZE & ZE & ZE & ZE & ZE \\
\hline PM & PB & NS & PS & PS & PS & PS & PS \\
\hline PB & PB & PM & PM & PM & PS & PS & PB \\
\hline
\end{tabular}

\subsection{The Fuzzy Control Query Table}

According to the input variables e, $\Delta \mathrm{e}$, fuzzy control state table and the membership function curves, the value of fuzzy control is obtained by L.A.Zadeh fuzzy inference:

$$
\mathrm{C}=\mathrm{u}(\mathrm{z} 1) /(\mathrm{z} 1)+\mathrm{u}(\mathrm{z} 2) /(\mathrm{z} 2)+\ldots+\mathrm{u}(\mathrm{zi}) /(\mathrm{zi})+\ldots+\mathrm{u}(\mathrm{zn}) /(\mathrm{zn})
$$

Then the fuzzy control query table is obtained according to the fuzzy decision based on the principles of gravity method, of which the formula is:

$$
\Delta \mathrm{u}=\frac{\sum_{i=1}^{n} z_{i} \times u\left(z_{i}\right)}{\sum_{i=1}^{n} u\left(z_{i}\right)}
$$

$Z_{i}$ is the element of the fuzzy control fuzzy drawn of inference. $u\left(z_{i}\right)$ is the degree of membership of fuzzy linguistic variables for $\mathrm{z}_{\mathrm{i}} \mathrm{n}$ is the number of fuzzy control elements drawn of fuzzy inference.

The adjusting of PID parameter must take the relationship the three parameters at different moment into account,which make controlled object achieve the best combination of static and dynamic performance. Then the fuzzy control rule table is established. The core of fuzzy control design is to establish a suitable fuzzy rule table based on the technical knowledge and practical experience of engineer and technical staff. The Inquiry table of output value is obtained according to the fuzzy rule table and the membership function of three output value $\Delta \mathrm{k}_{\mathrm{p}}, \Delta \mathrm{k}_{\mathrm{i}}, \Delta \mathrm{k}_{\mathrm{d}}$. According to the different $\mathrm{e}$ and $\Delta \mathrm{e}$, the amended parameters of output must to be find out and take it into equation (3). Then the correction of the PID parameters could be completed online.

$$
\begin{aligned}
& \mathrm{k}_{\mathrm{p}}=\mathrm{k}_{\mathrm{p}}{ }^{\prime}+\left\{\mathrm{e}_{\mathrm{i}}, \Delta \mathrm{e}_{\mathrm{i}}\right\}_{\mathrm{p}} \\
& \mathrm{k}_{\mathrm{i}}=\mathrm{k}_{\mathrm{i}}{ }^{\prime}+\left\{\mathrm{e}_{\mathrm{i}}, \Delta \mathrm{e}_{\mathrm{i}}\right\}_{\mathrm{i}} \\
& \mathrm{k}_{\mathrm{d}}=\mathrm{k}_{\mathrm{d}}{ }^{\prime}+\left\{\mathrm{e}_{\mathrm{i}}, \Delta \mathrm{e}_{\mathrm{i}}\right\}_{\mathrm{d}}
\end{aligned}
$$

\section{Matlab Simulation}

Using the following motor for the experiment to simulation analysis: three-phase squirrel cage induction motor ${ }^{[5]}$, the rated power at $\mathrm{P}_{\mathrm{N}}=1100 \mathrm{~W}$, the rated voltage of $\mathrm{U}_{\mathrm{N}}=380 \mathrm{~V}$, the rated speed of $\mathrm{n}_{N}=1410 \mathrm{n} / \mathrm{min}$, the stator resistance $\mathrm{R}_{\mathrm{s}}=5.3 \Omega$, the stator inductance $\mathrm{L}_{\mathrm{s}}=0.258 \mathrm{H}$, the rotor resistance $\mathrm{R}_{\mathrm{r}}=5.3 \Omega$, the rotor inductance $\mathrm{L}_{\mathrm{r}}=0.258 \mathrm{H}$, the stator and rotor mutual inductance $\mathrm{L}_{\mathrm{m}}=0.255 \mathrm{H}$, the speed of a given initial value is $1400 \mathrm{r} / \mathrm{min}$, in the $25 \mathrm{~s}$, the speed of motor is reduced to $800 \mathrm{r} / \mathrm{min}$.

Here are the control response curves of induction in motor speed regulation, respectively, using the traditional PID control and fuzzy adaptive PID (Figure 4, Figure 5) 


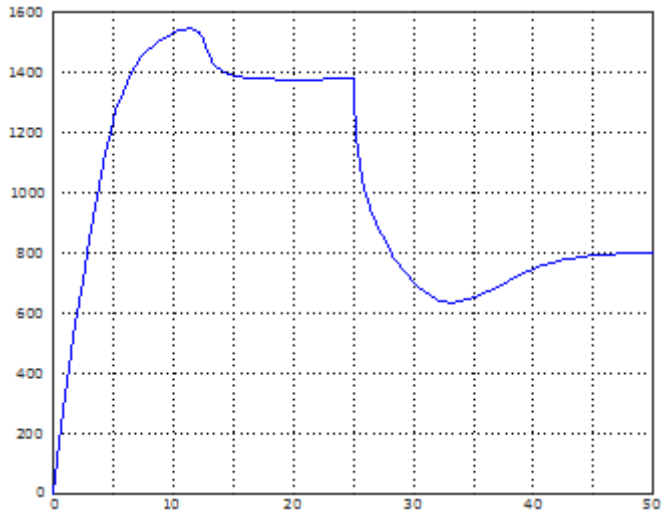

Figure 5.1. The traditional PID controller

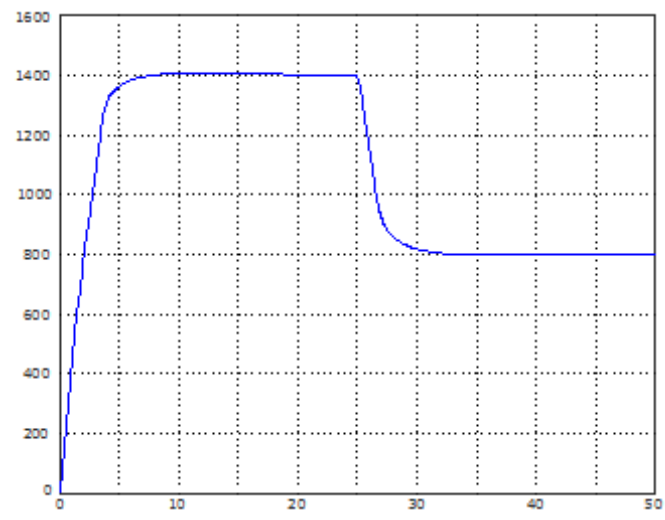

Figure 5.2. The fuzzy PID controller

\section{Conclusion}

The simulation results show that the regulation of the expected operating point of the motor in the motor test, the traditional PID controller is slower than the fuzzy PID controller in the adjusting of speed. And there is the phenomenon of overshoot. The tested motor could run quickly and accurately to the desired operating point using fuzzy PID controller, and there is no overshoot.

\section{References}

[1]. Finley W.R ,Hodowanec M.M and Hussain K.S 2003 Proper selection of induction motor tests IEEE 2003. 9-20.

[2]. Cai Jiagang and Wu Yaqi 2011 Motor testing technology and equipment manual (2nd edition) Mechanical Industry Press.

[3]. Kaiyuan Cai and Lei Zhang 2008 Fuzzy reasoning as a control Problem IEEE 2008. 600-614.

[4]. Liu Jinkun 2005 Intelligent Control Electronics Industry Press.

[5]. Ruan Yi and Chen Weijun 2006 Motion Control System Tsinghua University Press. 